\title{
PERFIL EPIDEMIOLÓGICO DE LA DISCAPACIDAD POR ACCIDENTES DE TRÁNSITO EN EL PERÚ, 2012
}

\author{
César Gutiérrez ${ }^{1, a}$, Franco Romaní2,b, Paolo Wong-Chero ${ }^{3, b}$, Juan José Montenegro-Idrogo 4,b
}

\begin{abstract}
RESUMEN
Objetivos. Describir el perfil epidemiológico de las personas discapacitadas por accidentes de tránsito (AT) en Perú. Materiales y métodos. Análisis secundario de la Encuesta Nacional Especializada Sobre Discapacidad (ENEDIS) del año 2012. Además, se realizó un análisis ecológico con los registros de AT del Ministerio de Transportes y Comunicaciones. Resultados. 49036 personas reportaron algún tipo de discapacidad por accidentes de tránsito (DAT); 81,3\% de los discapacitados reside en zonas urbanas. La discapacidad reportada más frecuente fue limitación en la locomoción y destreza $(77,4 \%)$, seguida de la discapacidad visual $(22,9 \%)$. Se reporta dependencia en $44,7 \%$ de las personas con discapacidad. Las regiones con mayor prevalencia de AT presentan mayor prevalencia de discapacidad por accidente de tránsito (Coeficiente de Spearman: 0,426, $p=0,034$ ). Conclusiones. La mayor parte de los discapacitados por AT proceden de la zona urbana, son varones y se encuentran en la edad económicamente productiva. La forma más común de limitación es la de locomoción. Gran parte de los afectados no reciben ningún tipo de rehabilitación, lo que acentúa la inequidad en salud relacionada a los accidentes de tránsito.
\end{abstract}

Palabras clave: Accidentes de tránsito; Personas con discapacidad; Prevalencia (fuente: BIREME).

\section{EPIDEMIOLOGICAL PROFILE OF TRAFFIC-RELATED DISABILITY IN PERU, 2012}

\begin{abstract}
Objectives. To describe the epidemiological profile of people living with disabilities due to traffic accidents (TA) in Peru. Materials and methods. Secondary analysis of the National Survey Specialized on Disability (ENEDIS) of 2012 and an ecological analysis of TA records of the Ministry of Transportation and Communications was done. Results. Disability by traffic accidents (DAT) was reported by 49,036 persons; $81.3 \%$ of whom live in urban areas. The most frequent disability was limited locomotion and skill $(77.4 \%)$, followed by visual impairment $(22.9 \%)$. Dependence for activities was reported in $44.7 \%$ of persons with disabilities. The regions with the highest prevalence of TA have a higher prevalence of disability by traffic accidents (Spearman coefficient: $0.426, p=0.034$ ). Conclusions. Most of disability due to TA is found in urban areas, correspond to males and consist of persons in economically productive age. The most common form of disability is in locomotion. Most of individuals do not receive any form of rehabilitation, which accentuates health inequity related to traffic accidents.
\end{abstract}

Key words: Accidents, traffic; Disabled persons; Prevalence (source: MeSH NLM).

\section{INTRODUCCIÓN}

Los accidentes de tránsito (AT) representan un grave problema de salud pública mundial, según la Organización Mundial de Salud más del $90 \%$ de las víctimas mortales por AT pertenece a países de ingresos bajos y medios quienes, paradójicamente, concentran solo el $48 \%$ de los vehículos del mundo. A nivel global cerca de 1,24 millones de personas mueren por AT cada año, además, se estima que se producen por año entre $20 \mathrm{a}$ 50 millones de traumatismo no mortales, siendo estas lesiones causa importante de discapacidad ${ }^{(1,2)}$.

En el Perú, la Estrategia Nacional de Accidentes de Tránsito del Ministerio de Salud reporta que en el quinquenio 2005-2009 se registraron más de 400 mil

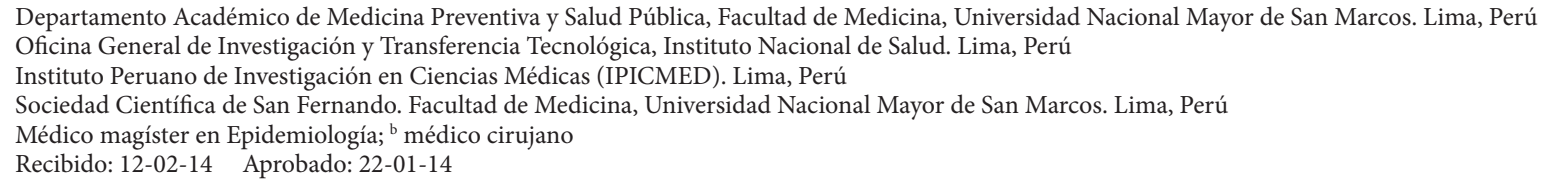

Citar como: Gutiérrez C, Romaní F, Wong-Chero P, Montenegro-Idrogo JJ. Perfil epidemiológico de la discapacidad por accidentes de tránsito en el Perú, 2012. Rev Peru Med Exp Salud Publica. 2014;31(2):267-73. 
accidentes de tránsito, con un saldo de casi dos mil muertos y cerca de 235 mil lesionados, más del $60 \%$ de los accidentes ocurrieron en Lima ${ }^{(3)}$. Por su parte, un estudio basado en una encuesta realizada en 2006, estimó que $2,93 \%$ de participantes reportaron estar involucrados en AT en el último año ${ }^{(4)}$. A pesar de las cifras indicadas, resulta difícil conocer la verdadera magnitud de los AT, debido, entre otras cosas, a la complejidad del problema y a la inexistencia de un sistema de registro adecuado y confiable ${ }^{(4)}$. Para aproximarnos al impacto de los AT en la población es necesario tener en cuenta una serie de variables que van desde los factores asociados a la ocurrencia del evento en sí, hasta la mortalidad o secuelas que dejan en la sociedad. Uno de los elementos más importantes que evaluar en la población sobreviviente es la carga de discapacidad (2).

En el Perú es escasa la evidencia de costos y discapacidad relacionados con AT. Un estudio en el Hospital Cayetano Heredia identificó 2001 pacientes que acudieron con lesiones por AT, de los cuales cerca de la mitad fueron los ocupantes del vehículo y la otra mitad peatones. En este estudio más de la mitad de los casos graves y casos mortales estuvo asociada al tipo de accidentes tránsito - peatón, con costos directos calculados en más de un millón y medio de soles (más de medio millón de dólares americanos) con similar cantidad estimada en los costos indirectos ${ }^{(5)}$. En España se ha documentado similar comportamiento en relación a carga de enfermedad por discapacidad por accidente de tránsito (DAT) ${ }^{(6)}$.

Los datos y cálculos en torno al dimensionamiento del impacto de los AT son difíciles de obtener en forma directa. Por ejemplo, el número y tipo de discapacidad generalmente reportada se reduce a aquellos pacientes que requieren intervención hospitalaria por lesiones graves, con un subregistro de aquellas personas con algún tipo de discapacidad que no requieren hospitalización (7). Este estudio buscó describir el perfil epidemiológico de las personas discapacitadas producto de AT, estimar su prevalencia a nivel nacional y por regiones, y determinar su relación con la prevalencia de AT, tomando como fuente la Encuesta Nacional Especializada Sobre Discapacidad.

\section{MATERIALES Y MÉTODOS}

\section{DISEÑO DEL ESTUDIO}

Este estudio corresponde a un análisis secundario de la Encuesta Nacional Especializada sobre Discapacidad (ENEDIS) del año 2012. La ejecución de la ENEDIS estuvo a cargo del Instituto Nacional de Estadística e
Informática (INEI), en coordinación con el Ministerio de la Mujer y el Consejo Nacional para la Integración de la Persona con Discapacidad (CONADIS). La ENEDIS 2012 fue una encuesta transversal que tuvo por objetivo: Obtener información estadística confiable sobre el tamaño de la población con alguna discapacidad existente en el país, el tipo de discapacidad que los afecta, sus características sociodemográficas y económicas; su nivel de funcionamiento personal, familiar y sociolaboral, con la finalidad de orientar adecuadamente las políticas, planes y programas para su atención ${ }^{(8)}$.

El diseño muestral de la ENEDIS corresponde a una muestra probabilística, estratificada, de áreas, bietápica e independiente en cada ámbito de inferencia (nivel nacional, regional, urbano/rural, costa/sierra/selva, Lima Metropolitana, Callao y Región Lima). El marco muestral fue elaborado con la información del XI Censo Nacional de Población y VI Censo Nacional de Vivienda del año 2007. Se realizaron entrevistas directas con personal capacitado del INEI en las viviendas seleccionadas, durante los meses de octubre a diciembre de $2012{ }^{(8)}$. Las bases de datos de los diferentes módulos de la ENEDIS 2012 se encuentran disponibles en el portal del servicio "Microdatos del INEI" (9).

\section{VARIABLES}

Para el presente reporte se seleccionó solamente a los entrevistados que para la pregunta 428 del cuestionario 02 (¿Cuál es el origen de esta(s) limitación(es)?) respondieron la opción 7 (accidente de tránsito). Las variables sociodemográficas empleadas para la descripción de la muestra fueron sexo, edad (en grupos decenales), nivel educativo, región natural (costa, sierra o selva), región política y zona de residencia (urbana o rural). La caracterización de la discapacidad se realizó a partir de las variables incluidas en la sección 400 del cuestionario 02: tipo de limitación (locomoción y destreza, visual, voz y habla, auditiva, intelecto, y conducta y relaciones interpersonales), la severidad de la limitación, el tiempo de discapacidad y el grado de autonomía y dependencia. También se analizó si los entrevistados recibían tratamiento o terapias de rehabilitación (pregunta 612 del cuestionario 02).

De manera complementaria, bajo una aproximación de un análisis de estudio ecológico de grupos múltiples, se recabó información sobre el número de AT fatales y no fatales según región para el año 2012, a partir de las estadísticas del Ministerio de Transportes y Comunicaciones ${ }^{(10)}$, de la población estimada a nivel regional por parte de INEI para el mismo año así como el tamaño del parque automotor por región para el año 2011, también obtenida del INEI (11). 


\section{ANÁLISIS ESTADÍSTICO}

Se realizó la descripción de las características de las personas con DAT mediante la elaboración de tablas de frecuencias absolutas y porcentuales, para variables cuantitativas se obtuvo la mediana y el intervalo intercuartil. El análisis se realizó tomando en cuenta los factores de expansión de la ENEDIS. Se estimó la prevalencia de DAT del año 2012 para cada región por cada 10 mil habitantes (número de personas que reportan como origen de discapacidad un accidente de tránsito/población estimada para el año 2012) y la prevalencia de AT del año 2012 para cada región por cada 10 mil habitantes (número de AT fatales y no fatales según región/población estimada para el año 2012).

Para correlacionar la prevalencia de DAT con la prevalencia de AT y el tamaño del parque automotor, se calculó el coeficiente de correlación de Spearman, previamente, se comprobó que dichas variables no seguían una distribución normal mediante la prueba de Kolmorogov-Smirnov. Se consideró como significativo un $p<0,05$. Para los análisis estadísticos se empleó el programa estadístico SPSS 15.0.

\section{ASPECTOS ÉTICOS}

La base de datos de la ENEDIS está disponible de manera gratuita para su uso por investigadores, y no cuenta con identificadores que permitan ubicar a los participantes, garantizando la confidencialidad de los datos. Al ser un estudio a partir de fuentes secundarias de uso público, en el cual no se están afectando los principios de protección a los participantes humanos en investigaciones, no fue necesaria la aprobación por un comité de ética.

\section{RESULTADOS}

\section{CARACTERISTICAS GENERALES DE DISCAPACITADOS POR ACCIDENTE DE TRÁNSITO}

La ENEDIS 2012 estima que 49036 personas a nivel nacional reportaron algún tipo de DAT, de los cuales $31984(65,2 \%)$ son varones. El 80,7\% de los discapacitados por accidente de tránsito tenían entre 30 y 79 años. El 36,6\% de los discapacitados alcanzó educación primaria y el $34,9 \%$ educación secundaria. En total, se estima que $39863(81,3 \%)$ discapacitados residen en zonas urbanas, la mayoría de los cuales se encuentra en la costa urbana $(n=28545,58,2 \%)$ (Tabla 1).
Tabla 1. Características sociodemográficas de personas con DAT, Perú 2012

\begin{tabular}{|cc}
\hline \multicolumn{1}{c}{ Características } & Porcentaje \\
\hline Sexo & \\
\hline Masculino & 65,2 \\
\hline Femenino & 34,8 \\
\hline Grupo etario & \\
\hline 0 a 9 & 1,5 \\
\hline 10 a 19 & 2,9 \\
\hline 20 a 29 & 7,4 \\
\hline 30 a 39 & 10,3 \\
\hline 40 a 49 & 14,2 \\
\hline 50 a 59 & 18,8 \\
\hline 60 a 69 & 20,7 \\
\hline 70 a 79 & 16,6 \\
\hline 80 y más & 7,5 \\
\hline Nivel educativo & \\
\hline Sin nivel & 10,9 \\
\hline Educación inicial & 0,7 \\
\hline Educación primaria & 36,6 \\
\hline Educación secundaria & 34,9 \\
\hline Educación básica especial & 0,2 \\
\hline Superior no universitaria & 7,8 \\
\hline Superior universitaria & 8,2 \\
\hline No datos & 0,7 \\
\hline Región de procedencia & $3,6,2$ \\
\hline Costa urbana & 1,2 \\
\hline Costa rural & 13,6 \\
\hline Sierra urbana & 6,5 \\
\hline Sierra rural & 3,6 \\
\hline Selva urbana & \\
\hline Selva rural & \\
\hline
\end{tabular}

\section{CARACTERISTICAS DE LA DISCAPACIDAD POR ACCIDENTE DE TRÁNSITO}

En las personas discapacitadas por accidente de tránsito, la limitación en locomoción y destreza fue la forma más frecuente $(77,4 \%)$, seguida por limitaciones visuales $(22,9 \%)$ (Tabla 2 ).

Entre los que refirieron limitación en la locomoción y destreza, el $49,5 \%$ refiere que es de tipo moderada, $35,7 \%$ de tipo grave y $5,4 \%$ es completa. En los que refirieron limitación visual, el 51,9\% presenta limitación moderada, $19,0 \%$ grave y $8,2 \%$ la limitación es completa. Entre los que refirieron limitación de la voz y habla, el $32,1 \%$ presentó limitación moderada, $40,6 \%$ grave y el $5,8 \%$ completa. Entre los que reportaron discapacidad auditiva por accidente de tránsito, el 49,2\% presentó 
Tabla 2. Tipo de limitación causada por accidente de tránsito, Perú 2012

\begin{tabular}{lcc}
\hline \multicolumn{1}{c}{ Tipo de limitación } & $\begin{array}{c}\text { Frecuencia } \\
\text { expandida }\end{array}$ & Porcentaje \\
\hline Locomoción y destreza & 37950 & 77,4 \\
Visual & 11217 & 22,9 \\
Intelecto & 7311 & 14,9 \\
Auditiva & 6159 & 12,6 \\
Conducta & 4661 & 9,5 \\
Voz y habla & 3047 & 6,2 \\
\hline
\end{tabular}

limitación moderada, $20,0 \%$ grave y 1,6\% completa. Entre los que refirieron limitación en el intelecto, el 56,3\% tuvo limitación moderada, $17,4 \%$ grave y $1,8 \%$ completa.

La mediana del número de años con discapacidad locomotora fue de 7 años (intervalo intercuartil: 3-16 años); para discapacidad visual fue de 5 años (intervalo intercuartil: 2-12 años); para discapacidad de la voz y el habla fue de 6 años (intervalo intercuartil: 2-11 años); para discapacidad auditiva fue de 5 años (intervalo intercuartil: 2,42-10 años), y para discapacidad del intelecto y la conducta la mediana coincidió en 5 años (intervalo intercuartil: 2-11 años).

\section{DEPENDENCIA GENERADA POR TIPO DE DISCAPACIDAD}

Se estima que $21910(44,7 \%)$ personas con algún tipo de DAT dependen para sus actividades diarias de alguna persona. Los que presentan limitación de la voz y habla son aquellos que en mayor proporción dependen de otras personas por su discapacidad $(76,4 \%)$, seguido de los que tienen limitación en la conducta $(58,6 \%)$ e intelecto $(55,5 \%)$ (Tabla 3$)$.

Al analizar la proporción de dependencia por grupo etario se observa que la dependencia en los extremos de la vida es más frecuente, especialmente hasta antes de los 19 años. Además, existen diferencias en la proporción de dependencia por sexo según grupos de edad. En mujeres es más frecuente hasta los 39 años, sin embargo, entre 40 y 59 años, la proporción es mayor en varones (Figura 1).

Tabla 3. Proporción de dependencia de otras personas por tipo de discapacidad

\begin{tabular}{lc}
\hline Tipo de discapacidad & Porcentaje \\
\hline Voz y habla & 76,4 \\
Conducta & 58,6 \\
Intelecto & 55,5 \\
Locomoción y destreza & 50,4 \\
Visual & 39,8 \\
Auditiva & 34,9 \\
\hline
\end{tabular}

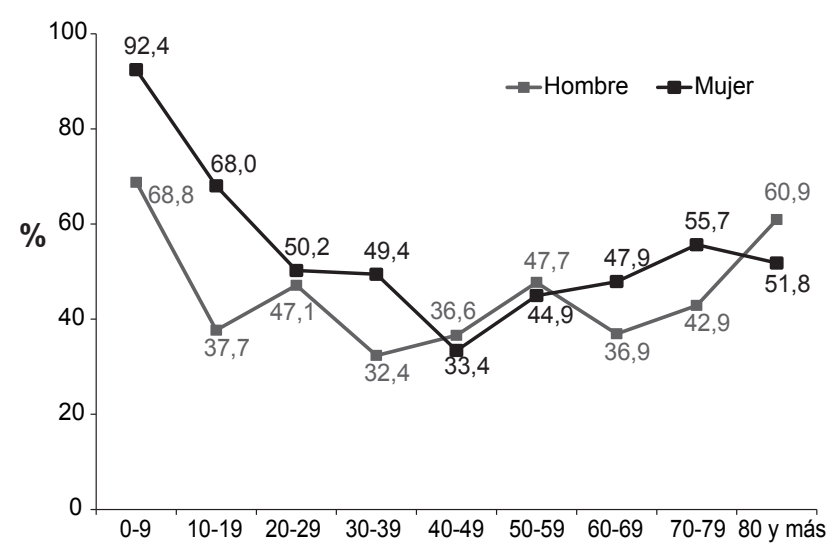

Figura 1. Proporción de dependencia por accidente de tránsito según grupo etario y sexo.

\section{DISCAPACIDAD POR ACCIDENTE DE TRÁNSITO Y ACCESO A REHABILITACIÓN}

Del total de personas discapacitadas por accidente de tránsito y que refirió necesitar algún tipo de rehabilitación, un estimado de 34350 (82,3\%) afectados debe recibir rehabilitación física, pero no la reciben. Esta proporción se incrementa hasta $99,9 \%$ para el caso de los que refieren necesitar terapia ocupacional, pero no la reciben (Tabla 4).

\section{PREVALENCIA ESTIMADA DE DISCAPACIDAD POR ACCIDENTE DE TRÁNSITO}

La mayor proporción de DAT corresponde a Lima $(39,5 \%)$, seguido de Puno (5,8\%) y Cusco (5,6\%). Respecto a la prevalencia estimada, en Tacna se estima 44,8 personas con DAT por cada 10000 personas, seguido de Moquegua (29,4/10 000 habitantes) y Tumbes (24,5/10 000 habitantes). Los departamentos con menor prevalencia fueron Amazonas (7,7/10 000 habitantes) y Loreto (6,5/10 000 habitantes). La prevalencia estimada a nivel nacional es de 16,3 personas con DAT por cada 10000 habitantes.

La prevalencia de DAT se encontró positivamente correlacionada con la prevalencia de AT a nivel de las regio-

Tabla 4. Discapacitados por accidentes de tránsito que necesitan algún tipo de rehabilitación y no la reciben

\begin{tabular}{lcc}
\hline \multicolumn{1}{c}{ Tipo de terapia } & $\begin{array}{c}\text { Personas que } \\
\text { refieren necesitar } \\
\text { rehabilitación }\end{array}$ & $\begin{array}{c}\text { Porcentaje que } \\
\text { no recibe la } \\
\text { rehabilitación }\end{array}$ \\
\hline Rehabilitación física & 34350 & 82,3 \\
\hline Tratamiento psicológico & 31084 & 96,6 \\
Apoyo emocional & 28369 & 99,3 \\
Terapia ocupacional & 27894 & 99,9 \\
Terapia de lenguaje & 18620 & 98,3 \\
Tratamiento psiquiátrico & 18550 & 98,7 \\
\hline
\end{tabular}


nes del Perú (coeficiente de Spearman: 0,426; $p=0,034$ ) (Figura 2). Las regiones con mayor prevalencia de AT presentan mayor prevalencia de DAT. No se encontró correlación entre el tamaño de parque automotor en las regiones con la prevalencia de DAT (coeficiente de Spearman: 0,113; $p=0,6$ ).

\section{DISCUSIÓN}

El presente estudio muestra tres importantes hallazgos, el primero es la correlación entre la prevalencia de AT y la prevalencia de personas con DAT por regiones; el segundo, la elevada proporción de dependencia en personas con DAT y, por último, la baja proporción de personas discapacitadas que necesitan y no reciben rehabilitación.

El análisis complementario usando un enfoque ecológico muestra que en las regiones con mayor prevalencia de AT se da una mayor prevalencia de DAT, si bien esta correlación puede ser evidente sin el presente análisis, brinda evidencia de la complejidad de este fenómeno y que no solo es necesario reducir la ocurrencia de AT sino también implementar acciones posaccidente. La magnitud de la discapacidad por accidente de tránsito se encuentra en función de la prevalencia de AT, de la gravedad del AT, y del acceso y respuesta de los servicios de salud. Por ello, la Organización Mundial de la Salud plantea cinco pilares que deben guiar los planes nacionales de seguridad vial, entre ellos el pilar cinco es la repuesta posaccidente ${ }^{(1)}$.

Aunque la finalidad debería ser prevenir los AT, muchas acciones pueden realizarse para minimizar el impacto de las lesiones producidas; siendo esencial la disponibilidad y eficiencia del cuidado prehospitalario y hospitalario. Un componente crítico que determina el resultado final de la persona que sufre un AT es la calidad de atención que recibe en el lapso entre el accidente y el acceso al cuidado de salud (1). La mayor proporción de personas con DAT proceden de zonas urbanas, especialmente de la costa, donde existe mayor acceso a los servicios de salud; sin embargo, la alta prevalencia de DAT en estas regiones sería consecuencia directa de la magnitud de los AT en dichas regiones y de sus características.

Entre las variables que podrían explicar la ocurrencia de AT y la gravedad de los daños físicos causados, se han propuesto: el volumen de vehículos de pasajeros (vehículos/hora); el volumen de vehículos pesados, y el volumen de vehículos ligeros (taxis, motocicletas) ${ }^{(12)}$. En una zona urbana de Irán, mediante un modelo matemático se concluye que el volumen de vehículos ligeros incrementa la probabilidad de accidentes más severos; los cuales son los que mayormente causan discapacidad de cualquier

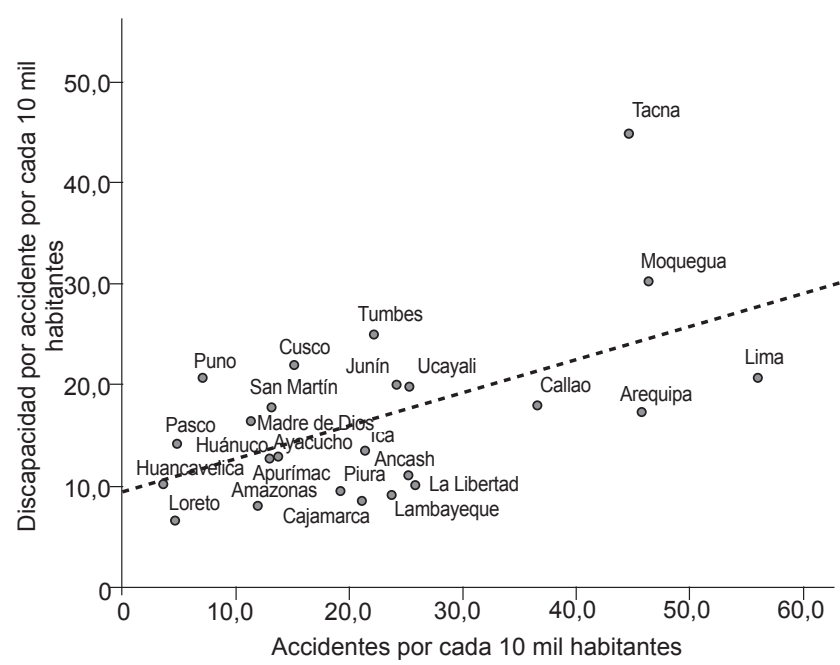

Figura 2. Correlación entre la prevalencia de accidente de tránsito y discapacidad por accidente de tránsito según regiones.

tipo. Por otro lado, el volumen de vehículos de pasajeros y de vehículos ligeros cumple una función importante en la probabilidad de accidentes que no causan daño físico a sus pasajeros. ${ }^{(12)}$ Otro estudio realizado en una zona urbana de bajo nivel socioeconómico de Lima, encontró que el elevado volumen de vehículos y su alta velocidad se encuentran asociados con lesiones por accidente de tránsito en niños peatones ${ }^{(13)}$.

Entre las características que se presenta en mayor proporción entre los DAT es el ser del sexo masculino $(65,2 \%)$, presentar nivel educativo secundario o menor $(83,2 \%)$ y tener entre 20 y 79 años $(88,1 \%)$. Este perfil es similar a lo reportado en otros estudios que caracterizan a los DAT; dichos estudios reportan que quienes muestran las siguientes características: ser varón, mayor de edad, residente de zona urbana, de menor nivel educativo y tener menor ingreso económico al mes, presentan mayor probabilidad de sufrir discapacidad por accidente de tránsito ${ }^{(14-16)}$.

En el presente estudio no fue posible analizar el nivel de ingreso mensual, lo cual permitiría realizar una mejor descripción del discapacitado por accidente de tránsito. Esta variable se encuentra relacionada con un mayor riesgo de discapacidad debido a que personas con menores niveles de ingreso son en mayor proporción peatones, ciclistas, son usuarios de buses de transporte público o motociclistas y, por otro lado, tienen limitado acceso a servicios de salud posaccidente lo cual incrementa la prevalencia de DAT.

El tipo de limitación más frecuente en el Perú, producida por AT, es la que afecta la locomoción y destreza, seguida en mucho menor proporción por limitaciones visuales, del 
intelecto y auditivas. Las lesiones en segmentos corporales que posteriormente producen algún tipo de limitación motora han sido reportadas como las más frecuentes en varios estudios en distintas regiones del mundo ${ }^{(6,17,18)}$.

Con respecto a los otros tipos de limitación, un estudio realizado en España encontró que 3,98\% de personas con DAT presentan dificultades para prestar atención visual o mantener atención auditiva; 4,16\% tiene dificultad para mostrar afecto o transmitir sentimientos, y $5,75 \%$ tiene dificultades para crear y mantener relaciones con amigos, vecinos y pares ${ }^{(6)}$. En nuestro estudio, el porcentaje de limitación en la conducta $(9,5 \%)$ e intelecto $(14,9 \%)$ entre los DAT es mayor.

Alrededor de siete años es el tiempo que los DAT presentan la limitación locomotora, para los otros tipos de limitación el tiempo varía entre 5 y 6 años hasta que desaparece. Esta característica define a los AT como un problema relacionado con la equidad. Es probable que la duración de las limitaciones que presentan las víctimas por AT no solo se deba a la gravedad del accidente sino también al pobre acceso a los servicios de salud, produciendo disparidades en la oportunidad de rehabilitación ${ }^{(19)}$. A nivel mundial, se estima que el efecto de daños no fatales en la pérdida de productividad es mucho mayor que el producido por lesiones fatales. Se estima que las pérdidas producidas por AT en las economías nacionales representan entre el uno y tres por ciento del producto bruto interno de un país por año; esta estimación incluye los costos médicos directos, indirectos y los costos a largo plazo ${ }^{(6,20,21)}$. El impacto económico derivado de la discapacidad y consecuente dependencia de los DAT es especialmente negativo para países en vías de desarrollo, debido a que los grupos de edad económicamente activos son los más vulnerables a dichas lesiones ${ }^{(16,20)}$.

El porcentaje de dependencia por DAT es variable según el tipo de limitación, el mayor porcentaje de dependencia se observa entre los que tienen limitación de la voz y habla $(76,4 \%)$, conducta $(58,6 \%)$, e intelecto $(55,5 \%)$. La mitad de los que tienen limitación de la locomoción son dependientes. La proporción de dependencia es mayor en los extremos de la vida. En el Perú, la prevalencia estimada de dependencia por DAT en la población general es de $0.16 \%$. Un estudio realizado en España calcula la prevalencia de dependencia por accidente de tránsito en $0,17 \%$, siendo mayor en las edades medias (entre 36 y 54 años) ${ }^{(6)}$. El mismo estudio describió la dependencia en tres grados (moderado, severo y total), lo cual permite establecer las demandas en servicios de salud y los costos en forma más exacta. En el presente análisis no se pudo determinar el grado de dependencia producido por AT.
Un aspecto alarmante de los resultados de este estudio, y que aporta más evidencia de las inequidades en salud en el país, es la baja proporción de personas con DAT que refieren necesitar rehabilitación y no la reciben. Un $82,3 \%$ de personas con DAT refirieron necesitar rehabilitación física, pero no la reciben, estos porcentajes superan el 95\% para los otros tipos de terapia, incluyendo tratamiento psicológico, apoyo emocional, terapia de lenguaje u ocupacional.

Se ha reportado que a los 6 meses pos-AT un 18\% de afectados tiene estrés postraumático ${ }^{(22)}$. Otro estudio encontró que al año del AT con trauma craneal la presencia de síntomas como cefalea, fatiga, vértigo, problemas de memoria, sensibilidad al ruido y dificultad en el pensamiento varía entre 3 y $16 \%{ }^{(23)}$. Esta evidencia pone de manifiesto la necesidad no solo de rehabilitación física, sino también la importancia del aspecto psicológico en el proceso de rehabilitación integral de una persona con DAT y que, según nuestros resultados, no vienen siendo abordados por el sistema de salud.

Entre las limitaciones del presente estudio, al ser un estudio de fuentes secundarias, existen variables que no pudieron ser exploradas como el ingreso mensual, la ocupación, el grado de dependencia entre otras que permitirían una mejor caracterización de persona con DAT. Por otro lado, las variables fueron medidas a través del autorreporte de las personas entrevistadas lo cual afecta la validez interna del estudio, por lo que el tipo de limitación, el grado de la limitación y la necesidad de rehabilitación, pueden no reflejar realmente el fenómeno estudiado. Se recomienda el cálculo de años de vida ajustado por discapacidad para estimar la magnitud del impacto a largo plazo de los AT.

En conclusión, se estima que a nivel nacional 49 036 personas viven con algún tipo de discapacidad por accidente de tránsito (prevalencia estimada de 16,3 por 10000 habitantes). Las regiones con mayor prevalencia de DAT son Tacna y Moquegua. Las lesiones causadas por los AT se ven potenciadas por la inequidad en salud existente; lo cual genera que entre el 82 y $99 \%$ de los discapacitados que refieren necesitar algún tipo de rehabilitación, no la reciben. Esto genera mayor dependencia que, dependiendo del tipo, puede llegar hasta el 76,4\% para el caso de discapacidad de la voz y habla; y $58,6 \%$ para el caso de discapacidad de conducta. Los resultados del presente estudio muestran que en las regiones con mayor prevalencia de AT existe mayor prevalencia de DAT, y que la población económicamente activa es la más afectada; dicha discapacidad, dependiendo del tipo de limitación, dura en promedio entre cinco a seis años. En vista de estos resultados son necesarios estudios que evalúen y caractericen directamente la presencia de DAT y los 
factores asociados a la gravedad de la limitación; además del análisis del impacto mediante años de vida ajustado por discapacidad y costo económico según región. Por otro lado, es necesario estudiar la oferta de servicios del sistema de salud (capacidad de respuesta) frente personas que sufren AT (atención inmediata) y para el manejo de las lesiones posteriores y consecuente rehabilitación.
Contribuciones de autoría: CGV, FRR participaron en la concepción y diseño del estudio, CGV participó en el análisis de datos, FRR y CGV participaron en la interpretación de los resultados, FRR, PWC,CGV y JMI participaron en la redacción del artículo, todos los autores aprobaron la versión final del manuscrito.

Fuentes de financiamiento: autofinanciado.

Conflictos de interés: los autores declaran que no tener conflictos de interés.

\section{REFERENCIAS BIBLIOGRÁFICAS}

1. World Health Organization. Global status report on road safety 2013: supporting a decade of action. Geneva: WHO; 2013.

2. Organitzación Mundial de la Salut. Informe sobre la situación mundial de la seguridad vial: es hora de pasar a la acción. Ginebra: OMS; 2009.

3. Choquehuanca-Vilca V, CárdenasGarcía F, Collazos-CarhuayJ, MendozaValladolid W. Perfil epidemiológico de los accidentes de tránsito en el Perú, 2005-2009. Rev Peru Med Exp Salud Publica. 2010;27(2):162-9.

4. Wong P, Gutiérrez C, Romaní F. Autorreporte de accidentes de tránsito en una encuesta nacional en la población urbana de Perú. Rev Peru Med Exp Salud Publica. 2010 Jun;27(2):170-8.

5. Bambarem Alatrista C. Características epidemiológicas y económicas de los casos de accidentes de tránsito atendidos en el Hospital Nacional Cayetano Heredia. Rev Medica Hered. 2004;15(1):30-6.

6. Alemany R, Ayuso M, Guillén M. Impact of road traffic injuries on disability rates and long-term care costs in Spain. Accid Anal Prev. 2013 Nov;60:95-102. doi: 10.1016/j. aap.2013.08.016.

7. Berecki-Gisolf J, Collie A, McClure R. Work disability after road traffic injury in a mixed population with and without hospitalisation. Accid Anal Prev. 2013 Mar;51:129-34. doi: 10.1016/j.aap.2012.11.010.

8. Instituto Nacional de Estadística e Informática. Encuestas y Registros: Encuesta Nacional Especializada sobre Discapacidad 2012 [Internet]. Lima: INEI [citado el 5 de enero de 2013]. Disponible en: http://www.inei.gob. pe/estadisticas/encuestas/

9. Instituto Nacional de Estadística e Informática. Microdatos: Bases de Datos [Internet]. Lima: INEI; 2012 [citado el 5 de enero de 2013]. Disponible en: http://iinei.inei.gob. pe/microdatos/

10. Perú, Ministerio de Transportes y Comunicaciones. Oficina de Estadística [Internet]. Lima: MTC [citado el 12 de agosto del 2013]. Disponible en: http://www.mtc.gob.pe/estadisticas/ index.html

11. Instituto Nacional de Estadística e Informática. Perú: Estimaciones y Proyecciones de Población Total por Sexo de las Principales Ciudades. 2000 -2015. Lima: INEI; 2012.

12. Ayati E, Abbasi E. Investigation on the role of traffic volume in accidents on urban highways. J Safety Res. 2011 Jun;42(3):209-14. doi: 10.1016/j. jsr.2011.03.006.

13. Donroe J, Tincopa M, Gilman RH, Brugge D, Moore DAJ. Pedestrian road traffic injuries in urban Peruvian children and adolescents: case control analyses of personal and environmental risk factors. PLoS One. 2008 Sep 10;3(9):e3166. doi: 10.1371/journal. pone.0003166.

14. Chakravarthy B, Anderson CL, Ludlow J, Lotfipour S, Vaca FE. The relationship of pedestrian injuries to socioeconomic characteristics in a large Southern California County. Traffic Inj Prev. 2010 Oct;11(5):508-13. doi: 10.1080/15389588.2010.497546.

15. Chen H, Du W, Li N, Chen G, Zheng $\mathrm{X}$. The socioeconomic inequality in traffic-related disability among Chinese adults: The application of concentration index. Accid Anal Prev. 2013 Jun;55:101-6. doi: 10.1016/j. aap.2013.02.025.

16. Loo BPY, Cheung WS, Yao S. The ruralurban divide in road safety: the case of China. Open Transp J. 2011;5:9-20.

17. Calil AM, Sallum EA, Domingues $C$ de
A, Nogueira L de S. Mapping injuries in traffic accident victims: a literature review. Rev Lat Am Enfermagem. 2009 Jan-Feb;17(1):120-5.

18. Dhondt S, Macharis C, Terryn N, Van Malderen F, Putman K. Health burden of road traffic accidents, an analysis of clinical data on disability and mortality exposure rates in Flanders and Brussels. Accid Anal Prev. 2013 Jan;50:659-66. doi: 10.1016/j.aap.2012.06.019.

19. Lapostolle A, Gadegbeku B, Ndiaye A, Amoros E, Chiron M, Spira A, et al. The burden of road traffic accidents in a French Departement: the description of the injuries and recent changes. BMC Public Health. 2009 Oct 13;9:386. doi: 10.1186/1471-2458-9-386.

20. Sharma BR. Road traffic injuries: A major global public health crisis. Public Health. 2008 Dec;122(12):1399-406. doi: 10.1016/j.puhe.2008.06.009.

21. Krug E. Decade of Action for Road Safety 2011-2020. Injury. 2012 Jan;43(1):6-7. doi: 10.1016/j. injury.2011.11.002.

22. Chossegros L, Hours M, Charnay P, Bernard M, Fort E, Boisson D, et al. Predictive factors of chronic posttraumatic stress disorder 6 months after a road traffic accident. Accid Anal Prev. 2011 Jan;43(1):471-7. doi: 10.1016/j. aap.2010.10.004.

23. Hours M, Chossegros L, Charnay P, Tardy H, Nhac-Vu H-T, Boisson $\mathrm{D}$, et al. Outcomes one year after a road accident: Results from the ESPARR cohort. Accid Anal Prev. 2013 Jan;50:92-102. doi: 10.1016/j. aap.2012.03.037.

Correspondencia: Franco Romani Romani Dirección: Instituto Nacional de Salud. Calle Cápac Yupanqui 1400, Jesús María-Lima Teléfono: 990630678

Correo electrónico:fromanir@gmail.com 